\section{Willy J. Malaisse}

\section{ADRESSE}

W.J. Malaisse : professeur de chimie pathologique. Laboratoire de médecine experimentale, faculté de médecine, université libre de Bruxelles, CP 618, campus Erasme, route de Lennik 808, 1070 Bruxelles, Belgique.

\title{
Anomalies du métabolisme insulaire dans le diabète
}

Différentes lésions du système glucosenseur des cellules $\beta$ du pancréas endocrine pourraient rendre compte des perturbations de la sécrétion d'insuline dans le diabète non insulinodépendant: déficit du GLUT-2, le transporteur membranaire du glucose; défaut de phosphorylation du glucose par mutation de la glucokinase ; excès d'activité de la glucose-6-phosphatase; déficit de la glycérophosphate déshydrogénase mitochondriale, l'enzyme clé de la navette du glycérophosphate; accumulation de glycogène insulaire. Des nutriments, capables de court-circuiter les étapes altérées du métabolisme glucidique, tels que les esthers méthyliques de l'acide succinique, pourraient donc, en principe, être utilisés pour stimuler la biosynthèse et la sécrétion d'insuline dans le diabète non insulinodépendant.

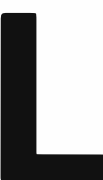

e progrès des connaissances dans le domaine de la sécrétion d'insuline au cours des trois dernières décennies a couvert cing étapes majeures. La régulation physiologique multifactorielle de cette sécrétion - tant par les nutriments circulants, les hormones et les neurotransmetteurs agissant de manière immédiate sur les cellules $\beta$ que par les facteurs ontogéniques, nutritionnels et endocriniens exerçant un contrôle à long terme fut d'abord établie [1]. Ensuite, l'axe cytophysiologique principal du processus sécrétoire, tel qu'il est déclenché par une élévation de la concentration extracellulaire en glucose, fut défini comme une séquence d'événements métaboliques, ioniques et mécaniques [2]. Le concept selon lequel l'identification du glucose et d'autres nutriments sécrétagogues par la cellule $\beta$ est étroitement liée à leur catabolisme dans les cellules insulaires justifia alors une étude détaillée de la régulation du métabolisme énergé- tique dans les îlots pancréatiques [3, 4]. Cette étude conduisit à son tour à la définition de lésions enzymatiques ou métaboliques responsables de l'insensibilité du pancréas endocrine au glucose dans les modèles de diabète non insulinodépendant $[5,6]$. Enfin, dans le prolongement de ces derniers travaux, des études furent récemment entreprises pour explorer une nouvelle approche thérapeutique fondée essentiellement sur l'utilisation de nutriments non glucidiques [7]. L'objet principal de cet article est d'évoquer certaines anomalies du métabolisme insulaire dans le diabète et leurs implications thérapeutiques. Les facteurs extrapancréatiques d'insulinorésistance ne sont pas pris en considération.

\section{Régulation \\ du métabolisme glucidique insulaire}

Le métabolisme du glucose dans les cellules productrices d'insuline pré- 


\section{RÉFÉRENCES}

1. Malaisse WJ. Hormonal and environmental modification of islet activity. In : Steine DF, Freinkel N, eds. Endocrine pancreas. Wa shington : American Physiological Society, 1972: $237-60$.

2. Malaisse WJ, Sener A, Herchuelz A, et al Sequential events in the process of glucoseinduced insulin release. In : Bajaj JS, ed. Diabetes. Amsterdam : Excerpta Medica, 1977 : 95-102.

3. Malaisse WI. Metabolic factors influencing synthesis and secretion of pancreatic islet hormones. In : Samols E, ed. The endocrine pancreas. New York : Raven Press, 1991 73-92.

4. Portha B. Physiologie de la cellule $\boldsymbol{\beta}$ des îlots de Langherans. médecine/sciences 1991 7 : 212-25.

5. Chanson P, Ferré $P$, Timsit J. Physiopathologie du diabète non insulinodépendant. médecine/sciences $1991 ; 7: 336-45$.

6. Malaisse WJ. Alteration of pancreatic $\beta$ cell D-glucose metabolism in type 2 diabetes : the G quintet. Endocrinologia 1993 ; $40: 309-13$.

7. Malaisse W. The beta cell in non-insulindependent diabetes : giving light to the blind. Diabetologia 1994 ; 37 (suppl 2) : 3642.

8. Unger RH. Diabetic hyperglycemia : link to impaired glucose transport in pancreatic $\beta$-cells. Science $1991 ; 251$ : 1200-5.

9. Thorens B, Wu Y], Leahy JL, Weir GC. The loss of GLUT 2 expression by glucose unresponsive $\beta$-cells of $\mathrm{db} / \mathrm{db}$ mice is reversible and is induced by the diabetic environment. J Clin Invest 1992 ; 90 : 77-85.

10. Thorens B, Weir GC, Leahy JL, Lodish HF. Reduced expression of the liver $/ \beta$ cell glucose transporter isoform in glucoseinsensitive pancreatic $\beta$-cells of diabetic rats. Proc Natl Acad Sci USA 1990 ; 87 : 6492-6.

11. Ohneda M, Johnson JH, Inman LR, $e$ al. GLUT-2 expression and function in $\beta$ cells of GK rats with NIDDM. Dissociation between reductions in glucose transport and glucose-stimulated insulin secretion Diabetes 1993 ; 42 : 1065-72.

12. Vionnet N, Stoffel M, Takeda J, et al Nonsense mutation in the glucokinase gene causes early-onset non-insulin-dependen diabetes mellitus. Nature 1992 ; 356 : 721-2.

13. Shimada F, Makino $\mathrm{H}$, Hashimoto $\mathrm{N}$, et al. Type 2 (non-insulin-dependent) diabetes mellitus associated with a mutation of the glucokinase gene in a Japanese family. Diabetologia 1993 ; $36: 433-7$.

14. Chiu KC, Tanizawa Y, Permutt MA. Glucokinase gene variants in the common form of NIDDM. Diabetes $1993 ; 42$ : 579-82.

15. Elbein SC, Hoffman M, Chiu K, et al. Linkage analysis of the glucokinase locus in familial Type 2 (non-insulin-dependent) diabetic pedigrees. Diabetologia 1993 ; 36 : sente plusieurs particularités qui contribuent à optimaliser la fonction glucorégulatrice de ces cellules. A cet égard, on peut citer les quatre exemples suivants $[3,4]$. D'abord, le passage du glucose au travers de la membrane plasmique des cellules $\beta$ se fait grâce à l'intervention d'un transporteur particulièrement efficace, le GLUT-2 $\left(m / s n^{\circ} 3\right.$, vol. 7, p. 291). Il en résulte un équilibre virtuellement immédiat et permanent des concentrations extracellulaire et intracellulaire de l'hexose. Ensuite, la phosphorylation du glucose en glucose-6-phosphate est catalysée non seulement par une hexokinase ubiquitaire, mais également par une glucokinase à faible affinité pour l'hexose. La constante de Michaelis apparente de la glucokinase pour le glucose est proche de $10 \mathrm{mM}$. La participation de cette enzyme à la phosphorylation du glucose permet donc d'augmenter la production de glucose-6-phosphate dans la gamme physiopathologique des concentrations glucidiques. Il convient de souligner, cependant, que la vitesse de phosphorylation de l'hexose est également influencée par une série d'autres facteurs, tels que la modulation de l'activité de la glucokinase par une protéine régulatrice, la liaison mitochondriale de la glucokinase, les modifications à long terme de la teneur insulaire en glucokinase, et la participation de l'ATP à une régulation synergique de type séquentiel. Dans les cellules insulaires, l'adaptation du flux glycolytique à la vitesse de phosphorylation du glucose met également en jeu l'activation de la phosphof ructokinase par des hexoses bisphosphates, tels que le glucose-1,6bisphosphate et le fructose-2,6-bisphosphate. De surcroît, la transformation par étape du glucose en fructose-1,6-bisphosphate semble également impliquer un processus de tunnelisation interenzymatique des intermédiaires glycolytiques. Enfin, une caractéristique essentielle du dispositif glucosenseur dans les cellules de Langerhans consiste en une stimulation préférentielle des processus d'oxydation mitochondriale par rapport au flux glycolytique global aux concentrations croissantes en glucose. Cette situation, plutôt exceptionnelle, est attribuable, en partie du moins, à l'activation par l'ion $\mathrm{Ca}^{2+} \mathrm{de}$ certaines déshydrogénases mitochondriales, telles que la FAD-glycérophosphate déshydrogénase, l'isocitrate déshydrogénase et le complexe de la 2-kétoglutarate déshydrogénase. Elle va de pair avec une stimulation préférentielle de la navette du glycérophosphate, de la décarboxylation oxydative du pyruvate et de l'oxydation dans le cycle de Krebs des résidus acétate produits à partir du glucose. Elle augmente considérablement le rendement de la production d'ATP couplée au catabolisme du glucose. Or, la vitesse de production de l'ATP représente précisément un facteur de liaison essentiel entre les événements métaboliques et cationiques dans la séquence sécrétoire.

\section{Anomalies métaboliques insulaires}

La perte de sensibilité au glucose des cellules $\beta$ dans les modèles de diabète non insulinodépendant pourrait s'expliquer par différentes lésions du système glucosenseur [3] (figure 1). Dans une revue récente, cing de ces lésions furent présentées [6] : (1) un déficit du GLUT-2 pourrait compromettre dans certaines cellules insulaires l'équilibre rapide des concentrations extracellulaire et intracellulaire du glucose $\left(m / s n^{\circ} 3\right.$, vol. 7, p. 291, [8-11]) ; (2) une mutation du gène de la glucokinase a été impliquée dans le diabète de type non insulinodépendant survenant chez de jeunes adultes [12-19]. Les propriétés catalytiques de la glucokinase sont sévèrement altérées dans les mutants de cette enzyme ; (3) un excès d'activité de la glucose-6-phosphatase pourrait rendre compte d'une perte d'ATP par opération d'un cycle futile entre le glucose et le glucose-6-phosphate [19] ; (4) dans différents modèles animaux de diabète non insulinodépendant, soit acquis, soit héréditaire, un déficit de la FAD-glycérophosphate déshydrogénase mitochondriale (m-GDH) fut observé dans les homogénats insulaires [20]. Ce déficit enzymatique ne se retrouve pas dans les tissus extrapancréatiques des animaux diabétiques. On peut également l'observer dans des îlots fraîchement isolés de patients diabétiques non insulinodépendants [21]; (5) le phénomène secondaire de glucotoxicité insulaire, 


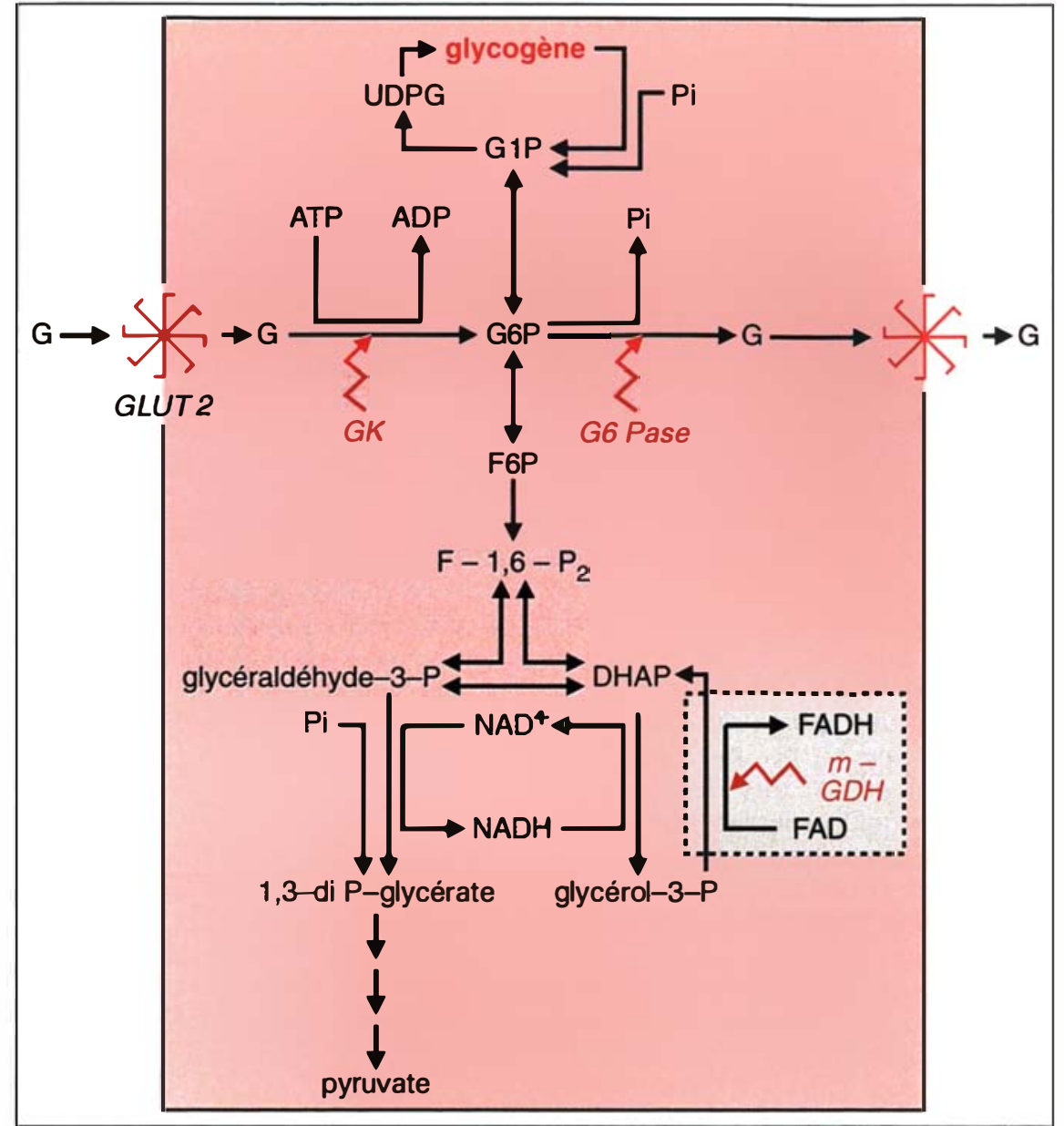

qui est la conséquence de l'hyperglycémie chronique dans le diabète, semble mettre en jeu une accumulation pathologique de glycogène dans les cellules insulaires. Dans les cellules chargées de glycogène, l'interférence de la glycogénolyse avec la glycolyse insulaire pourrait rendre compte de deux aspects typiques du phénomène de glucotoxicité insulaire, à savoir l'inhibition paradoxale et transitoire de la sécrétion d'insuline et la perte de sa spécificité anomérique, en réponse à l'administration rapide de glucose par voie intraveineuse.

Cette liste n'est pas exhaustive. D'autres lésions métaboliques ont été impliquées dans la genèse du diabète de type 2, telle une mutation de l'ADN mitochondrial [22]. De plus, un syndrome diabétique peut résulter d'anomalies acquises ou héréditaires dans le fonctionnement de la cellu$m / s n^{\circ} 2$, vol. 11, férier 95 le $\beta$, autres que celles frappant son métabolisme énergétique. Par exemple, il en va ainsi dans les anomalies de conversion de la pro-insuline en insuline [23] ou dans les perturbations de l'appareil microtubulaire des cellules $\beta$ [24]. Cependant, dans ces dernières situations, on imagine que le déficit sécrétoire ne concerne plus spécifiquement la seule riposte au glucose.

\section{Implications thérapeutiques}

Si l'on devait admettre que le diabète non insulinodépendant peut souvent être attribué à une lésion du système glucosenseur dans les cellules productrices d'insuline, il serait tentant de proposer que d'autres nutriments sécrétagogues capables de court-circuiter les étapes altérées du métabolisme glucidique puissent être utilisés
Figure 1. Vue schématique du métabolisme du glucose (G) dans la cellule $\beta$ des îlots pancréatiques. Le rectangle gris en traits interrompus encadre des événements mitochondriaux. Ce schéma attire l'attention sur 5 sites de perturbation du métabolisme glucidique insulaire dans le diabète non insulinodépendant, à savoir l'accumulation de glycogène, la carence en GLUT-2, une mutation de la glucokinase (GK), une activité excessive de la glucose-6-phosphatase (G6Pase) et un déficit en glycérophosphate déshydrogénase mitochondriale (m-GDH).

pour stimuler la biosynthèse de proinsuline et la sécrétion d'insuline dans le diabète de type 2 . C'est précisément dans cette perspective que des travaux récents ont porté sur l'utilisation des esters méthyliques de l'acide succinique comme agent insulinotrope. Alors que l'acide succinique exogène ne pénètre guère dans les cellules insulaires, ses esters méthyliques traversent efficacement la membrane plasmique et sont ensuite désestérifiés dans les cellules $\beta$. L'acide succinique devient ainsi disponible pour être métabolisé et, dès lors, pour stimuler tant la biosynthèse de pro-insuline que la synthèse d'insuline [25]. Les travaux menés à ce jour ont permis de mettre en évidence plusieurs avantages potentiels de cette approche thérapeutique nouvelle. Nous en citerons quelques exemples ; l'action insulinotrope de l'ester monométhylique (SAM) et di- 


\section{RÉFÉRENCES}

16. Tanizawa Y, Chiu KC, Province MA, et al. Two microsatellite repeat polymorphisms flanking opposite ends of the human glucokinase gene : use in haplotype analysis of Welsh Caucasians with Type 2 (non-insulindependent) diabetes mellitus. Diabetologia $1993 ; 36$ : 409-13.

17. McCarthy MI, Hitchins M, Hitman GA, et al. Positive association in the absence of linkage suggests a minor role for the glucokinase gene in the pathogenesis of Type 2 (non-insulin-dependent) diabetes mellitus amongst South Indians. Diabetologia 1993 ; $36: 633-41$.

18. Froguel $P$, Vionnet $N$, Gauguier $D$, Vaxillaire M, Zouali H, Passa P, Velho G. Génétique du diabète non insulinodépendant. médecine/sciences $1994 ; 10: 795-804$.

19. Khan A, Chandramouli V, Östenson CG, et al. Evidence for the presence of glucose cycling in pancreatic islets of the ob/ob mouse. J Biol Chem 1989 ; 264 : 9732-3.

20. Malaisse WJ. Is type 2 diabetes due to a deficiency of FAD-linked glycerophosphate dehydrogenase in pancreatic islets ? Acta Diabetol 1993 ; 30 : 1-5.

21. Fernandez-Alvarez J, Conget I, Rasschaert J, et al. Enzymatic, metabolic and secretory patterns in human islets of type 2 (non-insulin-dependent) patients. Diabetologia 1994 ; 37 : 177-81.

22. Kadowaki H, Tobe K, Mori Y, et al. Mitochondrial gene mutation and insulin-deficient type of diabetes mellitus. Lancet 1993 ; $341: 893-4$.

23. Chan SI, Nanjo $\mathrm{K}$, Miyano $\mathrm{M}$, et al. Abnormalities of insulin gene structure and expression - a minireview. In : SerranoRios M, Lefèbvre PJ, eds. Diabetes 1985. Amsterdam : Excerpta Medica, 1986 : 486-94.

24. Malaisse-Lagae F, Ravazzola M, Amherdt $M$, et al. An apparent abnormality of the $\beta$ cell microtubular system in spiny mice ( $A C O$ mys cahirinus). Diabetologia $1975 ; 11$ : 71-6.

25. Malaisse WJ, Rasschaert J, Villanueva-Penacarrillo ML, Valverde I. Respiratory, ionic, and functional effects of succinate esters in pancreatic islets. Am J Physiol 1993 ; $264: 428-33$.

26. Malaisse WJ, Sener A. Metabolic effects and fate of succinate esters in pancreatic islets. Am J Physiol 1993 ; 264 : 434-40.

27. Vicent D, Villanueva-Penacarrillo ML, Valverde I, Malaisse WJ. Enhancement of the insulinotropic action of glibenclamide by succinic acid methyl ester in anaesthetized rats. Med Sci Res 1993 ; 21 : 517-8.

28. Leclercq-Meyer V, Malaisse WJ. Enhancement by succinic acid dimethyl ester of insulin release evoked by D-glucose and glimepiride in the perfused pancreas of normoglycemic and hyperglycemic rats. Bio- méthylique (SAD) de l'acide succinique peut être attribuée à une aug mentation concertée de l'influx de l'acide succinique et de l'acétyl coenzyme A dans le cycle de Krebs. Le SAM et le SAD peuvent donc courtcircuiter les anomalies dans le transport du glucose, sa phosphorylation et son métabolisme ultérieur [26]. Le SAM conserve sa potentialité insulinotrope dans les modèles expérimentaux de diabète où le glucose a perdu sa capacité de stimuler la sécrétion d'insuline et provoque une inhibition paradoxale du débit insulinique [7]. Le SAM et le SAD potentialisent l'action insulinotrope des sulfamides hypoglycémiants [27]. Le SAD augmente de manière appréciable la sécrétion d'insuline par le pancréas isolé et perfusé, même à des concentrations glucidiques très élevées et même en présence de sulfamides hypoglycémiants [28]. De plus, une telle situation se retrouve même lorsque le pancréas est prélevé à des rats perfusés pendant deux jours avec une solution hypertonique de glucose, ce dispositif expérimental étant couramment utilisé comme modèle de glucotoxicité insulaire [28]. L'action insulinotrope du SAM et du SAD contraste avec l'absence de tout effet glucagonotrope de ces esters [27]. Enfin, les esters méthyliques de l'acide succinique ne représentent qu'un exemple parmi de nombreuses molécules apparentées susceptibles d'agir selon la même modalité. Par exemple, nous avons récemment montré que l'ester diméthylique de l'acide glutamique représente également un agent insulinotrope pleinement efficace.

\section{Conclusions}

On peut donc conclure cet article en pensant que des progrès substantiels peuvent être envisagés dans un futur proche, tant en termes de l'identification de nouvelles causes de déficit du système glucosenseur dans les cellules productrices d'insuline, qu'en termes d'outils nouveaux pour courtcircuiter de telles anomalies dans le diabète non insulinodépendant.

\section{TIRÉS À PART}

W.J. Malaisse.

\section{Summary}

Perturbation of islet metabolism in diabetes

Selected perturbations of pancreatic islet metabolism in diabetes are presented. Some possible therapeutical implications are also discussed, with emphasis on the use of non-glucidic nutrient secretagogues.

\section{Remerciements}

Le travail expérimental évoqué dans cet article a bénéficié de l'octroi de subsides du Fonds de la Recherche Scientifique Médicale. Je remercie Mme C. Demesmaeker pour son aide secrétariale. 